\title{
AN INFLUENCE OF ANTENNA TILT ANGLE TO THE RPW/SOLAR ORBITER DIRECTION FINDING
}

\author{
V. Krupar*, M. Maksimovic ${ }^{\dagger}$, O. Santolik ${ }^{\ddagger}$, and B. Cecconi ${ }^{\dagger}$
}

\section{Extended Abstract}

Solar Orbiter is an M-class mission in the ESA Science Programme Cosmic Vision 2015 - 2025 having an orbit with perihelion as low as 0.28 AU. The Radio Plasma Waves (RPW) Analyzer on board will provide new insights into the microscale phenomenon, the propagation modes of the radio waves and the localization of their source regions. The three electric antennas (each 5 meters long) are designed to be mounted on booms in a perpendicular plane to the spacecraft-Sun axis. Effective antenna lengths and directions are different from the physical ones due to their coupling with the spacecraft body. These parameters have been investigated considering various antenna placements on the spacecraft body [Rucker et al., this issue]. Results indicate that all effective antenna directions will be slightly tilted towards the Sun.

This paper discusses a possible accuracy of the Direction Finding (DF) with respect to this tilt angle and uncertainties of the effective antenna parameters. We have modeled spectral matrices that represent an unpolarized wave (Stokes parameters: $Q=0, U=0$, and $V=0$ ) propagating from a point source located on the Sun (an apparent source size: $\gamma=0^{\circ}$, a wave vector direction: $\theta=90^{\circ}$, and $\phi=$ $90^{\circ}$ ) as modeled by Cecconi [2007]. Effective antenna lengths have been assumed to be equal $\left(h_{1}=h_{2}=h_{3}\right)$, while effective antenna directions are varying with respect to the tilt angle $\left(\xi_{1}=0^{\circ}, \xi_{2}=-125^{\circ}\right.$, and $\left.\xi_{3}=125^{\circ} ; \zeta_{1}=\zeta_{2}=\zeta_{3}=90^{\circ}-65^{\circ}\right)$. We have performed various simulations for each set of parameters considering different sources of errors. The wave vector directions have been calculated using the Singular Value Decomposition method [Santolík et al., 2003; Krupar et al., 2010]. Figure 1a displays a mean difference in estimation of the wave vector directions (deviations from the Sun direction) as a function of the tilt angle $\zeta$. We have used normal distributions of lengths centered on the nominal lengths $h_{j}$ with sigma $\left(h_{j}\right) / h_{j}=$ $0.01,0.03$, and 0.05 (dotted, dashed, and solid line, respectively). An error analysis for uncertainty of the effective antenna direction is contained in Figure 1b. We have considered normal distributions of absolute deviations centered on the nominal

\footnotetext{
* Faculty of Mathematics and Physics, Charles University, 12116 Prague 2, Czech Republic

${ }^{\dagger}$ LESIA, Observatoire de Paris, UMR CNRS 8109, 92195 Meudon, France

$\ddagger$ Institute of Atmospheric Physics, Academy of Sciences of the Czech Republic, 14131 Prague 4, Czech Republic
} 


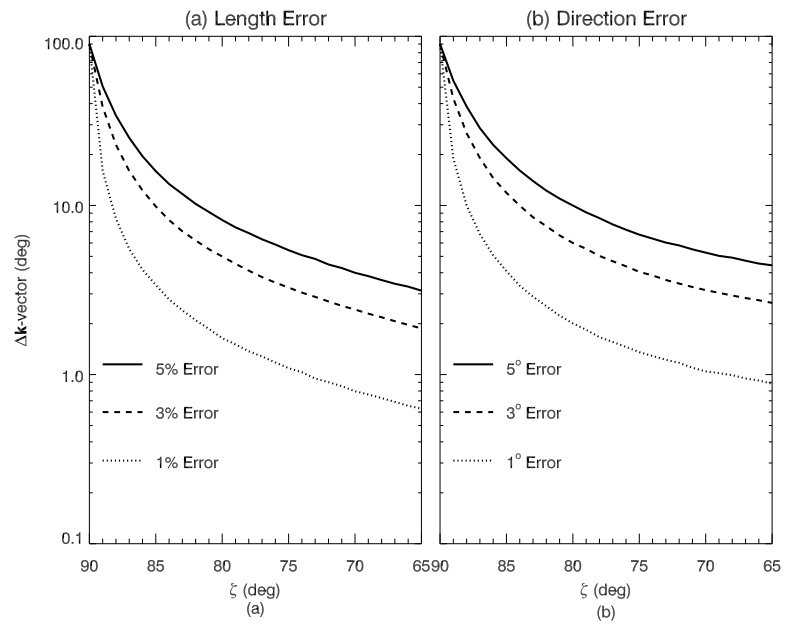

Figure 1: The average difference between input and output wave vector direction as a function of antenna tilt angle $\zeta$.

directions, with $\operatorname{sigma}\left(h_{j}\right) / h_{j}=0.01,0.03$, and 0.05 (dotted, dashed, and solid line, respectively) and uniform distributions of azimuth.

Our results indicate that we need the effective antenna tilt angle of at least $5^{\circ}-10^{\circ}$ to obtain the DF accuracy better than $10^{\circ}$ with reasonable experimental uncertainties.

\section{References}

Cecconi, B., Influence of an extended source on goniopolarimetry (or direction finding) with Cassini and Solar Terrestrial Relations Observatory radio receivers, Rad. Sci., 42, RS2003, 2007.

Krupar, V., M. Maksimovic, O. Santolik, B. Cecconi, Q. N. Nguyen, S. Hoang, and K. Goetz, The apparent source size of type III radio bursts: Preliminary results by the STEREO/WAVES instruments, 12th Int. Solar wind conference, AIP Conf. Proc., 1216, 284-287, 2010.

Rucker, H. O., M. Sampl, M. Panchenko, T. Oswald, D. Plettemeier, M. Maksimovic, and W. Macher, Implications of Antenna System Calibration on Spacecraft Design and Radio Data Analysis, in Planetary Radio Emissions VII, edited by H. O. Rucker, W.S. Kurth, P. Louarn, and G. Fischer, Austrian Acadademy of Sciences Press, Vienna, this issue, 475-485, 2011.

Santolík, O., M. Parrot, and Lefeuvre, F., Singular value decomposition methods for wave propagation analysis, Rad. Sci., 38, 1, 2003. 